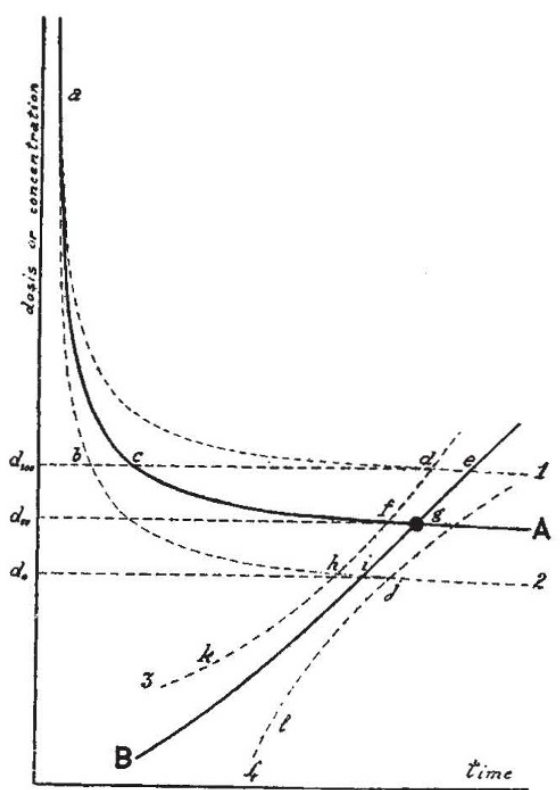

duration line $B$. Only for dosages between $d_{0}$ and $d_{100}$ does the individual variation play a part, effectors of resistance less than 50 per cent corresponding to the space $b c f h$, whereas points representing higher resistant effectors lie in deih. No representative points are comprised in the space $c f d$ or at the right side of egij, unless they exceed the limits of confidence, in which case, however, they may be neglected.

It is clear that the point $g$ represents the minimum effective dose for effectors of mean sensitivity, for example, the 50 per cent variation in any function connected with a particular stage in a graded action, or the L.D. 50 if curves of mortality in a population of small organisms are concerned. In the case of a skewed distribution of variation, the relative distance of hyperbolæ $1,2-3,4$ from the median curves $A$ and $B$ changes; but the general shape of the graph remains similar and its heuristic value unchanged.

The foregoing enables us to determine the point $g$ with an extremely small number of assays, since the development of the whole Trevan curve, giving the exact percentage of action for each dose between $d_{0}$ and $d_{100}$, becomes superfluous. Using the points lying in the neighbourhood of $a-c$ for calculating the hyperbola. (after substituting the reciprocal value of the survival times to convert the hyperbola in a straight line, and then employing the reciprocal value of the constants obtained to recalculate the reaction curve $A$ ), and those of the region $g k l$ for the duration line $B$, the degree of accuracy generally obtained with a score or so of animals is very unlikely to be further improved by increasing the number of assays to a hundred or more.

I have tested this method with digilanides on the frog heart to study detoxication-rates, with antihistaminic compounds on guinea pigs for quantitative measurements of antagonism, with mixtures of methyl donors and acceptors in order to control transmethylation-rates in mice, and in several other cases, with very satisfactory results.

Department of Pharmacology,

E. BECCARI

University of Ferrara. Sept. 26.

\section{Possible Influence of Inbreeding on Evolution}

According to Zeuner ${ }^{1}$ one of the difficulties of explaining evolution by the mutation theory is to account for the fact that elephants, which have the slowest rate of breeding, have had the fastest rate of evolution.

Those species producing the greatest number of generations in a given time might reasonably be expected to give rise to the greatest number of mutations and therefore to evolve the most rapidly. A possible solution of this anomaly has occurred to me.

Nearly all the mutations which might be of sig. nificance for evolution are recessive. Recent research ${ }^{2}$ has suggested that certain recessive genes can exert some influence on the survival prospects of individuals carrying them in the heterozygous condition. But it would seem that any factor which increases the chances for advantageous recessive mutations to appear in the homozygous state and thereby expose their full expression to the influence of natural selection should accelerate the rate of evolution.

The most effective factor of this kind is inbreeding, and elephants practise it to a considerable extent. Wild herds are served by a few dominant bulls which appear to mate regularly with their own daughters and are often succeeded by their sons. At the same time, owing to the migrations of young males, there is some interchange of genes between neighbouring herds. The elephant stocks, being split up into groups of semi-isolated colonies, therefore seem to provide the condition which, according to Sewall Wright ${ }^{3}$, is most favourable for evolution.

If the increased rate of evolution of the elephants has been due to the rapid exposure of recessive mutations by inbreeding in this way we might expect to find a large number of divergent species among ancestral forms.

The fossil record supports this contention. More than three hundred extinct species of elephants are known : only two species are living.

The same argument would seem to apply to deer, which practise a similar kind of group inbreeding and have had an exceptionally rapid rate of evolution. It may also have applied to wild horses and possibly to rhinoceroses. Keith ${ }^{4}$ has argued with considerable cogency the case for the importance of inbreeding in the development of the anthropoid apes and early men.

If some measure of evolutionary advantage be conceded to inbreeding, it is possible to ascribe 'biological sense' to certain phenomena which would otherwise seem to have little meaning.

There seems, for example, to be little other advantage in the remarkably developed homing behaviour of the salmon. It has been established that spawning Atlantic salmon return not only to the river of their birth but often to the very creek in which they were hatched. This must certainly result in a degree of inbreeding which would not occur otherwise.

The same argument may be applicable to the migration of storks, swallows and other birds which regularly return to the same neighbourhood to nest.

Daily Express, CHAPMAN PINCHER

Fleet Street, London, E.C.4.

1 Zeuner, F. E., Discovery (Aug. 1946).

2 Science, 108 (Nov. 12, 1948).

${ }^{3}$ Wright, Sewall, Proceedings of the VI International Congress of Genetics, 1 (1932).

"Keith, A., "A New Theory of Human Fvolution" (Watts and Co., 1948). 